\title{
UNIVERSAL FEATURES IN THE LAWS OF GROWTH
}

\author{
P.P. DELSANTO ${ }^{1}$, A. GLIOZZI ${ }^{1}$, D. ALEXANDRU IORDACHE ${ }^{2} \&$ C. GUIOT ${ }^{3}$ \\ ${ }^{1}$ Department of Physics, Politecnico di Torino, C.so Duca degli Abruzzi 24, 10129 Torino, Italy. \\ ${ }^{2}$ Department of Physics, University "Politechnica” of Bucharest, Splaiul Independenţei 313, Bucharest, Romania. \\ ${ }^{3}$ Department of Neuroscience, Università di Torino, C.so Raffaello 30, 10125 Torino, Italy.
}

\begin{abstract}
Computational models and simulations can be powerful tools for gaining an insight into the complex world of the biological mechanisms. Among them the growth processes are perhaps the most challenging and elusive to describe, for the different time scales and species specific features involved. Using a recently proposed technique for the analysis of experimental datasets (the Phenomenological Universalities Approach: PUN), we have succeeded to reproduce, to an excellent level of reliability, many results from experimental 'in vitro' and 'in vivo' tumor growth studies (e.g. the 'multipassaged' tumors described in the paper). Also the description of human growth, with its important implications for monitoring children development and diagnosing metabolic diseases, can be approached using the PUN method.

Keywords: best fitting, biological models, complexity, human growth, metabolic diseases, nonlinearity, phenomenological universalities, scaling, simulations, tumor growth.
\end{abstract}

\section{INTRODUCTION}

Growth of living beings and biological entities (e.g. tumors) has been extensively studied in the past. Most of the proposed laws are based on phenomenology, and simply fit experimental data in a (more or less) satisfactory manner.

More recently, some efforts have been devoted to find a scientific basis (mainly related to physics) for the proposed algorithms. In particular, West and collaborators have published their allometric model based on both the principle of energy conservation and the assumption that the development of a proper vascular network is the basis for growth in all living beings [1,2] and its main limiting factor, explaining growth saturation.

For most living organisms the vascular network develops according to some 'optimality principles' [3]: provided eight assumptions are done, regarding network scaling and hierarchical structure, constant branching ratio and space-filling properties and assuming that the network should minimize the energy losses and maximize exchange surfaces.

The growth law can be written as

$$
\mathrm{d} V / \mathrm{d} t=a V^{3 / 4}-b V
$$

where $V$ is the volume and $a$ and $b$ are suitable parameters. Note that the term $V^{3 / 4}$ can be interpreted as an 'effective exchange surface', e.g. in the case of a cell assuming nutrients only by diffusion through its membrane $S=V^{2 / 3}$. This formalism has been extended by Guiot et al. [4] for tumors.

Another very important application is human growth. Height, weight and body mass index (BMI) growth charts are universally adopted to monitor children growth and diagnose metabolic diseases, and are the basis of the field of auxology. Human growth has been described by a plethora of different algorithms, many of which simply aiming at best fitting average data [5].

\section{PHENOMENOLOGICAL UNIVERSALITIES}

Mathematical relationships, such as algebraic equations, ordinary differential equations (ODE), partial differential equations (PDE), etc. can be considered as true universalities, since their validity is 
completely independent from the field of application, be it physics, biology, economics or any other science. They are, however, 'a priori' (or 'top-down' [6]) universalities, even if they are the ultimate result of a considerable amount of experimental or observational work, since they no longer contain any reference to the experiments and observations from which they have been inferred. In other words, they are stated as valid or applicable 'per se' and often include the effect of intellectual manipulations, such as generalizations or corrections due to analogies, intuition or formal elegance. Their usefulness may be immediately appreciated in any kind of 'top-down' approach, in which they are applied to the solution of the most diverse problems in all fields of science.

In the present context we are concerned with Phenomenological Universalities (PUN), which represent an attempt to classify in well defined classes of universality datasets collected in all fields of science, based on simple unifying criteria. Thus, apart from their epistemologic interest, PUN's allow to set up a 'bottom-up approach, in which 'laws' or 'models' are inferred 'a posteriori', i.e. directly from data.

To be more specific, we define PUN's as a tool for solving the following problem:

Given the string of data $z_{i}=z\left(t_{i}\right),(i=1, \ldots, I)$ with $t$ being the time (or any other suitable variable), then:

1. Find a best fitting function $z(t)$, based not on a given numerical procedure, but on a general formalism independent of the field of application.

2. From the fitting function obtain a model for the proposed phenomenology.

The above problem can be easily extended to vectorial datasets, such as $z_{k i}=z_{k}\left(t_{i}\right),(k=1, \ldots, K)$, in which correlations exist among several variables $z_{k}$, all functions of the same independent variable $t$, which might also be vectorialized. In the case $K=2$, the use of a complex notation both for the variables and the parameters, allows to use, for the solution of the problem, the same formalism developed in the following sections.

\subsection{Procedure}

Assuming for brevity to have a single dataset $\mathrm{z}\left(t_{i}\right)$, the problem defined above can be solved by searching for a relationship of the kind

$$
G^{N}\left(a, b, p_{1}, p_{2}, \ldots, p_{N}\right)=0
$$

which represents the truncation of a suitable expansion to the $N$-th term, with $N$ fitting parameters $p_{n}$. In eqn (1) $a=\dot{z}, \quad b=\dot{a}=\ddot{z}$, with dots denoting differentiation with respect to the independent variable, i.e. $\dot{z}=\mathrm{d} z / \mathrm{d} t$. In general $a=a(z, t)$, but in the following we restrict ourselves to the case $a=a(z)$. The cases $a=a(t)$ and $a$ given by a sum of contributions from two terms, $\bar{a}(z)$ and $\tilde{a}(t)$, each depending on only one of the two variables, are discussed in Ref. [7].

As a simple but very important example, we consider the sub-case of eqn (2), in which $b=b(a)$. Since

$$
b=\dot{a}=\frac{\mathrm{d} a}{\mathrm{~d} z} \dot{z}=a \frac{\mathrm{d} a}{\mathrm{~d} z}
$$

assuming

$$
\frac{\mathrm{d} a}{\mathrm{~d} z}=\beta+\gamma a+\delta a^{2}+\ldots
$$


if we truncate the expansion at the $N$-th term, then we call the corresponding PUN class UN. It follows, in the case $N=2$ (i.e. U2):

$$
b=\sum_{n=1}^{2} \alpha_{n}^{(N)} a^{n}=\beta a+\gamma a^{2}
$$

Eqn (5), or its generalization to UN with $N>2$, has a very simple interpretation (or justification). Even if the experimentally measured quantity is $z(t)$, the relevant observable, for what concerns the evolution of the system, is $a$. In a linear approximation we would expect its derivative $b$ to be proportional to $a$, for reasons of scaling. But our overwhelming experience in all fields of science points out to a nonlinear Universe, with the linearity being just an approximation or a special (often ad hoc) case. Therefore we should also expect a nonlinear $b(a)$.

One could, of course, in addition to the 'space' $(a, b)$, consider others, such as $(z, a)$ or $(z, b)$, but we do not do it here, since they would lead to already well explored ODE's (or PDE's). Other PUN classes in the space $(a, b)$ may also be considered, e.g. the extended UN class EUN, for which

$$
b=\sum_{n=0}^{N} a_{n}^{(N)} a^{n}=\alpha+\beta a+\gamma a^{2}+\ldots
$$

or the VN class, defined by

$$
a=\sum_{n=1}^{N} \beta_{n}^{(N)} b^{n}
$$

The classes EUN will be considered in the next section.

Obviously such PUN classes are of interest only if some phenomenology described by them may be found. For example, EU2 seems to be able to represent the growth of successive implantations of tumoral lines in cavies or other lab animals.

\subsection{The class UN}

The class U1 and its correspondence with the well known (and most extensively used) Gompertz law are discussed in Ref. [8] Therefore in the following we limit ourselves to derive the basic formalism for the class U2, which includes, as special cases, the well known law for ontogenetic growth of West and collaborators [1-3] and its extension to tumoral growth [9]. From the differential equations $\dot{a}=b$ and eqn (5), it follows:

$$
a=a_{0}\left[(1+\psi) e^{-\beta t}-\psi\right]^{-1}
$$

where $\psi=(\gamma / \beta) a_{0}$ and $a_{0}=a(z(0))$. Then from $\dot{z}=a$ and $z(0)=0$, which does not represent a real restriction (since a translation can be performed), it easily follows:

$$
z=-\frac{1}{\gamma} \ln \left[1+\psi\left(1-e^{\beta t}\right)\right]
$$


A specially relevant case can be obtained by letting $z=\ln y$. Then we have the usual growth equation:

$$
\dot{y}=a(y) y
$$

with the solution

$$
y=\left[1+\psi\left(1-e^{\beta t}\right)\right]^{-1 / \gamma}
$$

It can be easily proven that $y$ satisfies the ODE

$$
c_{1} y^{p}=\frac{d y}{d t}+c_{2} y
$$

where $p=1+\gamma, c_{2}=\beta / \gamma, c_{1}=c_{2}(1+\psi)$.

In many applications (e.g. in biology, see [10]), a very important interpretation can be attached to eqn (12). That is, we may consider $c_{1} y^{p}$ as the energy input, $\mathrm{d} y / \mathrm{d} t$ the growth of the system and $c_{2} y$ the energy consumption (e.g. due to the metabolism). Then saturation (i.e. no further growth: $\mathrm{d} y / \mathrm{d} t=0)$ follows when $c_{1} y^{p}=c_{2} y$.

According to the 1st law expressed per unit time, any variation in the internal energy is related to the difference between the input energy (1st term of the RHS of eqn (1), accounting for the nutrient/ oxygen inlet from the surface by diffusion, or through the vascular network) and the energy required for metabolism, which is normally dependent on the volume of the system. Any positive variation in the internal energy can be stored in the system (e.g. by adipose mass) or, alternatively, used for growth (LHS of eqn (1)).

This interpretation, which implies that eqn (12) is an expression of the energy conservation of the system (or of the First Principle of Thermodynamics), is very intriguing, since it suggests that, as a direct result of the nonlinearity of eqn (5), fractality follows (since in general $p$ is not integer, unless the energy input is uniformly distributed over the whole system). If the energy enters the system through its external (e.g. spherical) surface, then $p=2 / 3$. In the model of West and collaborators $p=$ 3/4. In a recent model by Guiot et al. [11] the exponent $p$ varies between a first avascular phase of tumoral growth and the onset of angiogenesis.

It is interesting to remark that in the next PUN class U3, eqn (12) becomes

$$
c_{1} y^{p}=c_{2} \frac{d y}{d t}+c_{3} \frac{d y^{p}}{d t}-c_{4} y
$$

that is an additional term $c_{3}\left(\mathrm{~d} y^{p} / \mathrm{d} t\right)$ appears as a fractal-like dimensioned contribution to the growth. The coefficients $c_{1}, c_{2}, c_{3}$ and $c_{4}$ are not reported here for brevity.

From eqn (11), by defining

$$
u=y^{-\gamma}-1
$$

and

$$
\tau=1-e^{\beta t}
$$


it follows

$$
u=\psi \tau
$$

that is, the scaling invariance, which was lost in eqn (10), due to its nonlinearity, is regained by means of the adoption of the fractal-dimensioned variables $u$ and $\tau$.

\subsection{The class EUN}

In Section 2.1, eqn (6) may be 'justified' as a plausible generator of PUN classes on aesthetical grounds, since it completes the power expansion of $b(a)$, which in the UN formalism (see eqns 3-5) lacks the $n=0$ term $a$. A correlation between the classes EUM and $\mathrm{UN}$, with $\mathrm{N}=\mathrm{M}+1$, may be easily obtained by defining:

$$
f(a)=\sum_{n=0}^{M} a_{n} a^{n}=\alpha_{M} \prod_{n=1}^{M}\left(a-r_{n}\right)
$$

where $r_{n}$ are the roots of $f(a)$, including possibly multiple ones and complex conjugate (c.c.) pairs.

In the case $\mathrm{EUM}$, from $b(a)=f(a)=\mathrm{d} a / \mathrm{d} t$ it follows:

$$
t=t(a)=I=\int \frac{d a}{f(a)}
$$

whereas, in the case $\mathrm{UN}$, from $b(a)=a f(a)=\mathrm{d} a / \mathrm{d} t$ it follows:

$$
t=t(a)=J=\int \frac{d a}{a f(a)}
$$

The integral $I$ can be easily evaluated, starting from the formula [12]:

$$
\frac{1}{f(a)}=\sum_{n=1}^{M} \frac{1}{f^{\prime}\left(r_{n}\right)\left(a-r_{n}\right)}=\sum_{n=1}^{M} \frac{p_{n}}{a-r_{n}}
$$

where the prime denotes derivation with respect to $a$ and

$$
p_{n}=\left[f^{\prime}\left(r_{n}\right)\right]^{-1}
$$

It follows for EUM, apart from an integration constant:

$$
t(a)=\sum_{n=1}^{M} p_{n} \ln \left(a-r_{n}\right)=\ln \prod_{n=1}^{M}\left(a-r_{n}\right)^{p_{n}}
$$

For the evaluation of $J$ we can apply eqn (20) to the function $g(a)=a f(a)$ and let $n=0$, M with $r_{0}=0$. Then

$$
\frac{1}{a f(a)}=\frac{1}{a f(0)}+\sum_{n=1}^{M} \frac{q_{n}}{a-r_{n}}=\sum_{n=0}^{M} \frac{q_{n}}{a-r_{n}}
$$

where $q_{0}=1 / f(0)$ and $q_{n}=p_{n} / r_{n}$ for $n=1, M$. 
It follows for $\mathrm{UN}$ (with $N=M+1$ and apart from an integration constant):

$$
t(a)=\sum_{n=0}^{M} q_{n} \ln \left(a-r_{n}\right)=\ln \prod_{n=0}^{M}\left(a-r_{n}\right)^{q_{n}}
$$

It is important to recall that, in case of c.c. pairs of roots,

$$
\ln (x+i y)=\ln (r \exp (i \Phi))=\ln r+i \Phi
$$

where and $r=\sqrt{x^{2}+y^{2}} \Phi=\operatorname{arctg} y / x(0 \leq \Phi<\pi)$,

So that, instead of logarithms of complex numbers, it is possible to express the results in terms of $\operatorname{arctg}$ of real numbers [12, idem, eqn 2.172, p. 68].

In order to obtain the solution $z(t)$, or $y(t)=\exp z(t)$, one must integrate the ODE $\mathrm{d} z / \mathrm{d} t=a(t)$. The prerequisite for this, of course, is the inversion of the relationship $t(a)$, given by eqns (22) and (24) for EUM and UN, respectively.

This can be easily done up to $M$ or $N=2$. The results are summarized in Tables 1 and 2 for EUM and UN, respectively.

Table 1: Solutions for the PUN classes EUM, $M=0,1,2$.

\begin{tabular}{llll}
\hline \multicolumn{1}{c}{$b(a)$} & \multicolumn{1}{c}{$a(t)$} & \multicolumn{1}{c}{$z(t)$} \\
\hline EU0 & $a$ & $a t+a_{0}$ & $1 / 2 a t^{2}+a_{0} t$ \\
EU1 & $a+\beta a$ & $r+\left(a_{0}-r\right) B$ & $r t+1 / \beta\left(a_{0}-r\right)(B-1)$ \\
EU2 & $a+\beta a+\gamma a^{2}$ & $a_{0}(p-q E) /(1-E)$ & $p t-1 / \gamma \ln \left((1-E) /\left(1-E_{0}\right)\right)$ \\
\hline
\end{tabular}

The following notations have been used: $a_{0}=a(0) ; B=\operatorname{ext}(\beta t) ; E_{0}=(1-p) /(1-q) ; E=E_{0} \exp (\gamma$ $(p-q) t), r=\alpha / \beta, p$ and $q$ are the roots of the equation $b(a)=0$ for EU2.

Table 2: Solutions for the PUN classes UN, $N=0,1,2$.

\begin{tabular}{llll}
\hline & $b(a)$ & \multicolumn{1}{c}{$a(t)$} & \multicolumn{1}{c}{$z(t)$} \\
\hline $\mathrm{U} 0$ & 0 & $a_{0}$ & $a_{0} t$ \\
$\mathrm{U} 1$ & $\beta a$ & $a_{0} B$ & $a_{0}(B-1) / \beta$ \\
$\mathrm{U} 2$ & $\beta a+\gamma a^{2}$ & $a_{0} B /(1+\Phi-\Phi B)$ & $-1 / \gamma \ln (1+\Phi(1-B))$ \\
\hline
\end{tabular}

The following notations have been used: $a_{0}=a(0) ; B=\operatorname{ext}(\beta t) ; \Phi=\gamma a_{0} / \beta$. The reported solutions are, of course, in agreement with the ones found in Section 2.2.

Many plots obtained for U1, U2 (and its generalizations UN/TM, obtained when $a(t, z)=\bar{a}(t)+\breve{a}(z)$ ) may be found elsewhere [7]. No plots of EUM growth curves have been reported so far. Figure 1 accounts for the first four members of the family.

Therefore, in the next section, we present some representative examples, in the spirit of building up an 'Atlas of PUN classes' helping experimentalists to select a suitable class for novel datasets. We wish to recall that an extremely beautiful and comprehensive 'Atlas of Functions' [13] has been recently compiled for the 'mathematical universalities'. 

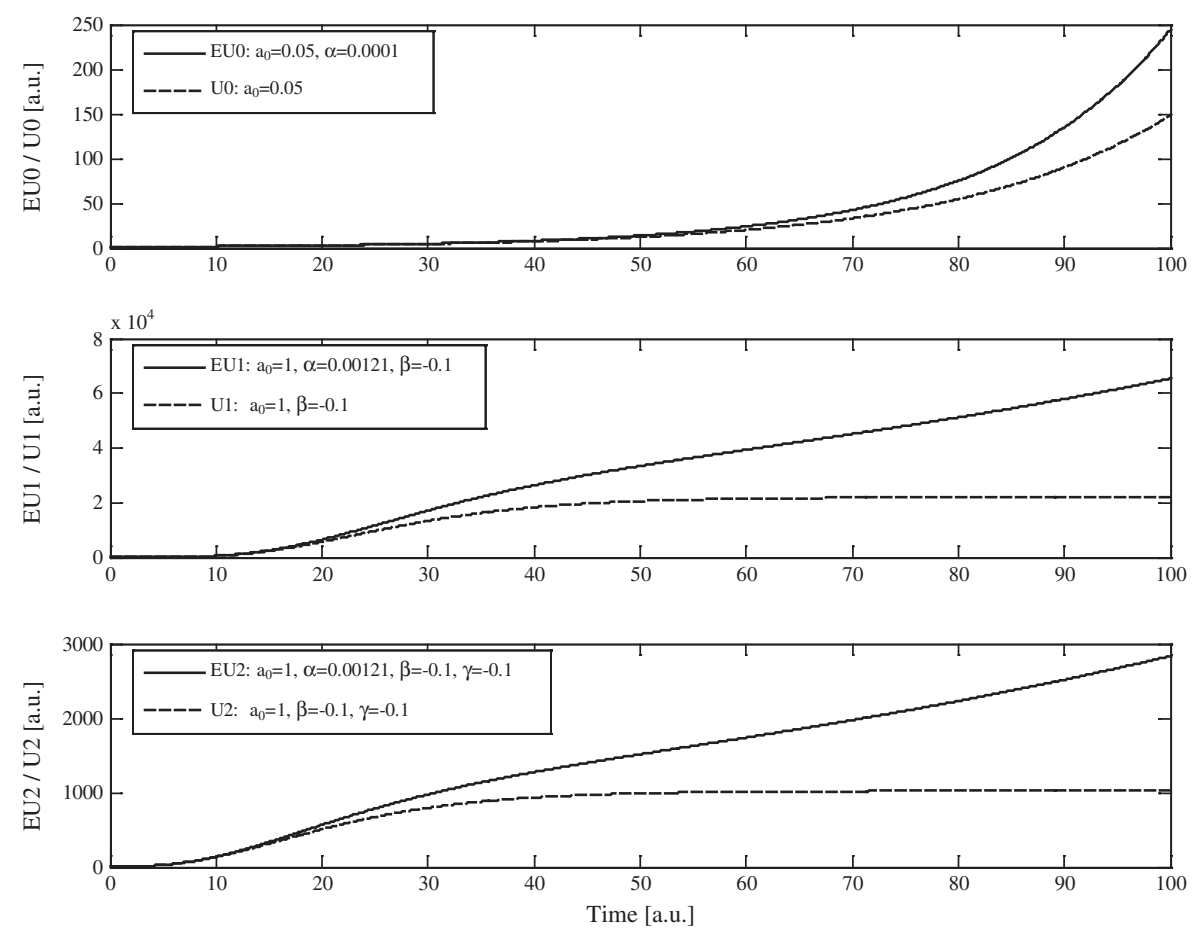

Figure 1: Some examples of different behaviors for the universal classes EUN and the corresponding class UN.

\section{NOTABLE APPLICATIONS OF THE PUN CLASSES UN: TUMOR GROWTH}

The PUN class U0 describes a simple exponential growth law, which properly applies to tumoral cells keeping on replicating with a constant duplication time in the absence of mechanical or nutritional restrictions. Contrary to expectations, such a model is not totally unrealistic. In fact, U0 represents the first phase of any 'in vitro' experiment, and also the initial phase of all tumors.

Such an unrestricted growth may last for days, months or even years provided a healthy, nutrient rich environment is available, as in the biological model of the Multi Passage Tumor (MPT), where tumors grow following the subcutaneous implantation on the back of a lab animal (usually mice) of $\sim 10^{6}$ tumor cells (from cell cultures or surgical resection). Tumor cells are then passaged from one mouse to another by harvesting them from a growing tumor and implanting a given number of them into another healthy animal. Once the tumor has grown above a certain volume, it is harvested again $[14,15]$. For example, McCredie et al. reported the case of a spontaneous mammary tumor in a $\mathrm{C} 3 \mathrm{H}$ mouse, from which the first syngenic transplant was done in 1946 and which has been serially transplanted into the $\mathrm{C} 3 \mathrm{H} / \mathrm{HeJ}$ strain, reaching in 1971 the 900th generation.

As shown in Figure 2, tumors grow at a larger rate in each successive transplant, i.e. the growth curves become progressively steeper, which can be explained by observing that, being the new seed reimplanted each time after a short time $T$ (e.g. 10 days) into a new healthy, nutrient rich environment; thus we can assume at each 'passage' an experimental growth law with approximately the same rate $c$. The tumor mass, after $n$ passages in which the same amount $m_{0}$ of tumor 

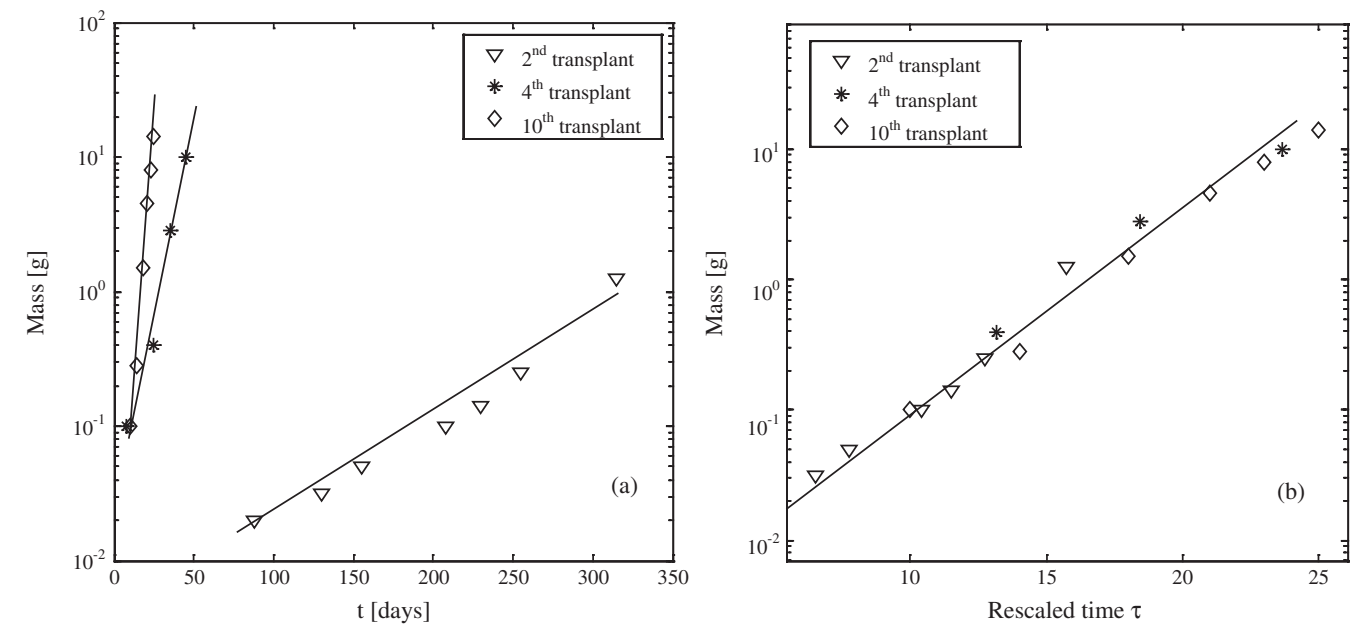

Figure 2: Results from Steel [14]. Up to 10 transplants of cells from the tumoral line rat fibroadenoma have been performed, but the curves corresponding to only three of them have been reported [9] in logarithmic scale. As discussed in the text, the averaged growth curves become increasingly steeper with successive passages, due to the aging of the newly transplanted tumor cells. By rescaling the time, we obtain a plot (b), in which all the curves are collapsed into a unique one.

is reimplanted, and at a time from the beginning of the whole procedure equal to $t=n T+\Delta t$, will be given by [17]:

$$
m(t)=m_{0}(1+\exp (n c T)(\exp (c \Delta t)-1))
$$

Eqn (25) shows that the exponential trend is corrected by a term, which accounts for the real age of the tumor and increases at each transplant, thus accelerating the growth.

If we follow the tumor growth curve for a longer time (even 'in vitro'), a necrotic core will develop, due to the 'screening' of the nutrients flow from the cells of the outer layers, so that a saturation level is reached. This corresponds to the PUN class U1, predicting a saturation value:

$$
V_{\infty}=V_{0} \exp \left(c_{0} / \beta\right)
$$

which is normally seen in MTS and is normally described using the Gompertz law [17]. Most aggressive tumors overcome nutrients deprivation by means of angiogenesis, and the neo-vascular network partly supports growth, as discussed by Guiot et al. [18], following the model of West and collaborators [1-3]. Often this third phase is complemented, in 'in vivo' or 'ex vivo' tumors by the processes of tumor invasion and metastasis. Starting from West's law (1), a generalized expression for growth is then:

$$
\mathrm{d} V / \mathrm{d} t=a S-b V
$$

where $S$ is the embedding layer acting as boundary with the host (i.e. the effective 'surface' from which nutrients come and waste products are removed), and $V$ the volume, to which the energy consumption for metabolism is assumed to be proportional. Note: $S$ is not in general a 2D surface. 
This stage of tumor growth corresponds to the PUN class U2. The class U2 includes, besides Gompertz as a special case, all the growth models proposed to date in all fields of research, i.e. besides the already mentioned model of West and collaborators [1-3], also the exponential, logistic, thetalogistic, potential, von Bertalanffy, etc. (see for a review De Vladar [19]).

The choice of the specific 'strategy' for maximizing the exchange surface depends on many factors, according to the characteristics of the host and the 'molecular weapons' available.

This encompasses the possibility that the tumor evolves by changing the 'degree of fractality' of its nutrient input system and may imply that the fractal parameter $p$ varies dynamically through tumor growth [11, 20], which requires resorting to the PUN class U3.

\section{NOTABLE APPLICATIONS OF THE PUN CLASSES EUN: HUMAN GROWTH}

Understanding human growth, and particularly some phases, such as the perinatal and the juvenile ones, has always been challenging. Simple curves based on well established assumptions, and widely applied to other fields (such as the Gompertz curve) are inadequate, and therefore purely phenomenological curves, selected on the basis of their 'fitting', were traditionally used.

Contrary to most mammals, the growth curve of some 'social' species, such as elephants, lions, primates and men, cannot be described by a simple curve, but requires at least two Gompertz- or logistic-like curves (or three for humans, see for instance [21]), describing the early growth and the juvenile phases separately.

According to Devenport [22], the human growth rate exhibits three maxima: one intrauterine, a second around the 6-th year and the third around the 16-th year, possibly activated by the secretions of the pituitary gland or the anterior lobe of the hypophysis.

In addition to the above main accelerations, many authors have pointed out that short-term oscillations are detectable looking at longitudinal data. In the classical paper of Butler et al. [23], 135 children were monitored at six monthly intervals from 2 to 18 years of age.

Longitudinal studies made apparent a cyclic, rhythmic pattern, as a cycle of spurts and lags occurring from the age of 2 until adolescence.

Actually, in the historical paper of Wales [5] even very short time cyclicities, such as the postural changes in height throughout the day, due to spinal disc compression, are reported. Variations in height velocity have been described with the season of the year, possibly modulated through the higher central nervous system and secretion of melatonin and other hormones with circadian rhythmicity.

All the above effects cannot obviously be deduced by phenomenologic models. Moreover, being the algorithms different and selected 'ad hoc', to compare the results from studies performed in different institutions, or between normal vs. pathological growth (normally described by different growth charts) is virtually impossible. The PUN model, and in particular the EUM family seems, on the contrary, to be able to describe the growth using few, unifying variables.

Figure 3 represents the various types of growth which can be found in human growth reported in the variables $\mathrm{d} z / \mathrm{d} t$ vs. $z$.

Actually, in many phases of human growth (as, for instance, fetal growth [24], see Figure 4) the relationship $a$ vs. $z$ exhibits some non-monotonicity, i.e. some 'spurts'. Contrary to UN, the EUN family, proved to account for such spurts.

The analysis of the obtained results (starting from some existing data concerning the human body growth) suggests that the use of the similitude 'phases space' $(z, \dot{z})$ allows the identification of the main types of growth stages, which can be reproduced by the EUM universality class.

Further work is needed to estimate the parameter values which best fit the various human growth phases and to better exploit all the model features, and possibly to explore its implications for 


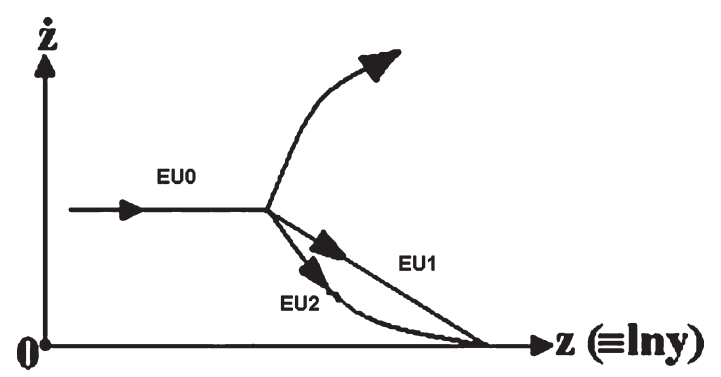

Figure 3: A sketch of the growth curves which can be found in various phases of human growth reproducible with the EU0, EU1 and EU2 models.

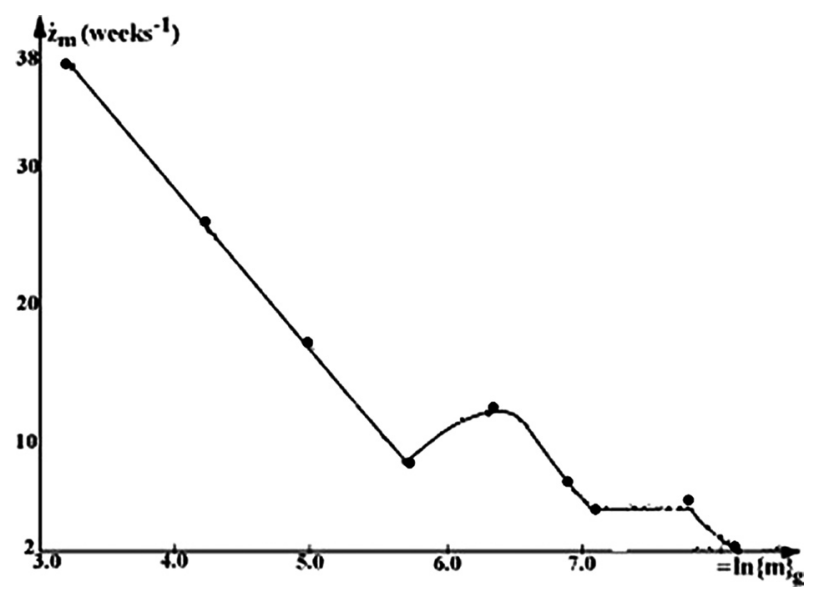

Figure 4: Fetal growth: sketch of the line connecting the experimental data (estimated mass reported in Ref. [24]). Data are represented using the logarithm of the mass on the $x$-axis and its time derivative on the $y$-axis).

diagnosing various growth pathologies. For instance, by assuming that different 'clones' of cells can be activated at different times (e.g. by hormones, such as GH or sexual hormones), the EUM curves can be further modulated to better resemble the realistic human growth curves.

By comparison between any specific individual growth curve and the collection of many representative ones, the numerical evaluation of the timing and the amplitude at the onset of the "growth spurts' could eventually lead to a better diagnostics of some hormonal disorders as well as to a better monitoring of the related therapies.

\section{ACKNOWLEDGEMENTS}

One of the authors (ASG) acknowledges the support of a Lagrange fellowship from the C.R.T. Foundation. CG kindly acknowledges the contribution from Regione Piemonte (Progetti Sanitari Finalizzati 2008) and Università di Torino.

\section{REFERENCES}

[1] West, G.B., Brown, J.H. \& Enquist, B.J., A general model for ontogenetic growth. Nature, 413, pp. 628-631, 2001. doi:10.1038/35098076 
[2] West, G.B. \& Brown, J.H., Life's universal scaling laws. Physics Today, 57, pp. 36-43, 2004. doi:10.1063/1.1809090

[3] Savage, V.M., Deeds, E.J. \& Fontana, W., Sizing up allometric scaling theory. PLoS Comput Biol, 4, pp. e1000171, 2008. doi:10.1371/journal.pcbi.1000171

[4] Guiot, C., Degiorgis, P.G., Delsanto, P.P., Gabriele, P. \& Deisboeck, T.S., Does tumor growth follow a "universal law"? J Theor Biol, 225, pp. 147-151, 2003. doi:10.1016/S0022-5193(03)00221-2

[5] Wales, J.K.H., A brief history of the study of human growth dynamics. Ann Human Biol, 25, pp. 175-184, 1998; see also Canessa, E., Modeling of body mass index by Newton's second law. J Theor Biol, 248, pp. 646-656, 2007.

[6] Bejan, A. \& Marden, J.H., Unifying constructal theory for scale effects in running, swimming and flying. J Exp Biol, 209, pp. 238-248, 2006. doi:10.1242/jeb.01974

[7] Delsanto, P.P., Gliozzi, A.S. \& Guiot, C., Scaling, growth and ciclicity in biology: a new computational approach. Theoretical Biology and Medical Modelling, 5, p. 5, 2008, doi:10.1186/17424682-5-5. doi:10.1186/1742-4682-5-5

[8] Delsanto, P.P., Gliozzi, A.S. \& Bosia, F., A comparison of different instances of phenomenological universalities, Proceedings of the 9th WSEAS International Conference on Mathematics \& Computers In Biology \& Chemistry, Bucharest, Romania, pp. 36-41, 2008.

[9] Delsanto, P.P., Griffa, M., Condat, C.A., Delsanto, S. \& Morra, L., Bridging the gap between mesoscopic and macroscopic models: the case of multicellular tumor spheroids. Phys. Rev. Lett., 94, pp. 148105, 2005.

[10] Castorina, P., Delsanto, P.P. \& Guiot, C., Classification scheme for phenomenological universalities in growth problems in physics and other sciences. Phys Rev Lett, 96, pp. 188701, 2006. doi:10.1103/PhysRevLett.96.188701

[11] Guiot, C., Delsanto, P.P., Carpinteri, A., Pugno, N., Mansury, Y. \& Deisboeck, T.S., The dynamic evolution of the power exponent in a universal growth model of tumors. $J$ Theor Biol, 240, pp. 459, 2006. doi:10.1016/j.jtbi.2005.10.006

[12] Gradshteyn, I.S. \& Ryzhik, I.M., Tables of Integrals, Series and Products, Academic Press: New York, pp. 56-7, 1980.

[13] Oldham, K., Myland, J. \& Spanier, J., An Atlas of Functions, Springer: New York, 2008.

[14] Steel, G.G., Growth Kinetics of Tumors, Clarendon Press: Oxford, 1977.

[15] McCredie, J.A. \& Sutherland, R.M., Differences in growth and morphology between the spontaneous $\mathrm{C} 3 \mathrm{H}$ mammary carcinoma in the mouse and its syngenic transplants. Cancer, 27, pp. 635642, 1971. doi:10.1002/1097-0142(197103)27:3<635::AID-CNCR2820270319>3.0.CO;2-F

[16] Gliozzi, A.S., Guiot, C. \& Delsanto, P.P., A new computational tool for the phenomenological analysis of multipassage tumor growth curves. PLoS ONE, 4, e5358, 2009, doi:10.1371/ journal.pone.0005358.

[17] Gompertz, B., On the nature of the function expressive of the law of human mortality and a new mode of determining life contingencies. Phil Trans $R$ Soc, 115, pp. 513-585, 1825. doi: $10.1098 /$ rstl.1825.0026

[18] Guiot, C., Pugno, N., Delsanto, P.P. \& Deisboeck, T.S., Physical aspects of cancer invasion. Phys. Biol., 4, P1-P6, 2007, doi:10.1088/1478-3975/4/4/P01.

[19] De Vladar, H.P., Density-dependence as a size-independent regulatory mechanism, $J$ Theor Biol, 238, pp. 245-256, 2006. doi:10.1016/j.jtbi.2005.05.014

[20] Delsanto, P., Gliozzi, A.S., Bruno, C.L.E., Pugno, N. \& Carpinteri, A., Scaling laws and fractality in the framework of a phenomenological approach. Chaos, Solitons \& Fractals, ISSN: 0960-0779, 2008. 
[21] Lozy, M.E., A critical analysis of the double and triple logistic growth curves. Ann Hum Biol, 5, pp. 389-394, 1978. doi:10.1080/03014467800003021

[22] Devenport, C.B., Human growth curve. J Gen Physiol, 10, pp. 205-216, 1926. doi:10.1085/ jgp.10.2.205

[23] Butler, G.E., McKie, M. \& Ratcliffe, S.G., The cyclical nature of prepubertal growth. Ann Hum Biol, 17, pp. 177-198, 1990. doi:10.1080/03014469000000952

[24] (a) http://embriology.med.unsw.edu.au/wwwhuman/Stages/Cstages.htm, (b) http://embriology. med.unsw.edu.au/wwwhuman/Stages/Cst800.jpg, (c) http://embriology.med.unsw.edu.au/ wwwhuman/Hum.10wk/Images/fetalweight.jpg. 